BMJ Open Sport \& Exercise Medicine

\title{
Study protocol of a 52-week Prospective Running INjury study in Gothenburg (SPRING)
}

\author{
Jonatan Jungmalm, ${ }^{1}$ Stefan Grau, ${ }^{1}$ Pia Desai, ${ }^{2}$ Jon Karlsson, ${ }^{2}$ \\ Rasmus Østergaard Nielsen ${ }^{3}$
}

To cite: Jungmalm J, Grau S, Desai P, et al. Study protocol of a 52-week Prospective Running INjury study in Gothenburg (SPRING). BMJ Open Sport \& Exercise Medicine 2018;4:e000394. doi:10.1136/ bmjsem-2018-000394

- Additional material is published online only. To view please visit the journal online (http://dx.doi.org/10.1136/10. 1136/bmjsem-2018-000394).

Accepted 29 May 2018
Check for updates

${ }^{1}$ Center for Health and Performance, Department of Food and Nutrition and Sport Science, University of Gothenburg, Gothenburg, Sweden

${ }^{2}$ Sahlgrenska Academy, Department of Orthopaedics, Sahlgrenska University Hospital, Gothenburg, Sweden ${ }^{3}$ Section of Sport Science, Department of Public Health, Aarhus University, Aarhus, Denmark

Correspondence to Jonatan Jungmalm; jonatan. jungmalm@gu.se

\section{ABSTRACT}

Introduction It is assumed that a running-related (overuse) injury occurs when a specific structure of the human body is exposed to a load that exceeds that structures' load capacity. Therefore, monitoring training load is an important key to understanding the development of a running-related injury. Additionally, other distribution, magnitude and capacity-related factors should be considered when aiming to understand the causal chain of injury development. This paper presents a study protocol for a prospective cohort study that aims to add comprehensive information on the aetiology of running-related injuries and present a new approach for investigating changes in training load with regard to running-related injuries.

Methods and analysis This study focused on recreational runners, that is, runners exposed to a minimum weekly average of $15 \mathrm{~km}$ for at least 1 year. Participants will undergo baseline tests consisting of a clinical/anthropometrical examination and biomechanical measurements. Furthermore, participants will log all training sessions in a diary on a weekly basis for 1 year. The primary exposure variable is changes in training load. A medical practitioner will examine runners suffering from running-related pain and, if possible, make a clear diagnosis. Finally, additional time-varying exposure variables will be included in the main analysis, whereas the analysis for the secondary purpose is based on timefixed baseline-related risk factors.

Ethics and dissemination Ethical approval (DNR: 712-15) for the study and its design was obtained from the Gothenburg regional ethical review board. The results of the study will be published in peer-reviewed journals.

\section{BACKGROUND}

Running is one of the most popular forms of physical exercise among able-bodied individuals on a global scale. ${ }^{1}$ Millions of people across Europe run regularly, with proportions reported as 29\% in Denmark, $25 \%$ in Germany, $19 \%$ in Belgium, $17 \%$ in Sweden, $14 \%$ in Finland and $12 \%$ in the Netherlands. ${ }^{2-5}$ Interestingly, the popularity of running has increased tremendously in the past decades, which is demonstrated in part by the growing amount of running events ${ }^{5}$ and increasing number of runners completing road races, such as $5 \mathrm{~km}, 10 \mathrm{~km}$, half-marathon and marathon distances. ${ }^{2}$ The increase in popularity is in contrast to other sports, for example soccer and handball, which have been experiencing a decline in the proportion of athletes over the last few years. ${ }^{36}$ Running is characterised by its nature of simplicity, the opportunity to exercise whenever and wherever suitable and the relatively low cost. These characteristics makes running advantageous to other sports requiring fixed training days and more expensive equipment. Major reasons for running as a primary exercise modality include, but are not limited to, increased physical and/or psychological well-being, weight loss, self-development, performance enhancement and social interaction. ${ }^{2} 7$ From a health perspective, physical exercise, including running, ${ }^{8}$ offers substantial benefits, such as reduced risk of several metabolic, cardiovascular, psychiatric, pulmonary and neurological diseases compared with a sedentary behaviour. ${ }^{9}$ Consequently, the public health gains of keeping runners active should not be underestimated. ${ }^{8}$

There are barriers that lead to a temporary or permanent restriction of running activity. These include injuries, illness, engagement in other social and physical activities, lack of time or motivation, as well as pregnancy and childcare. ${ }^{10}$ Of these, running-related injuries may be viewed as runners' primary enemy, since injuries are reported as the most common reason for men and the third most common reason for women to quit running over a 10 -year period. ${ }^{10}$ The negative impact that running-related injuries has on some individuals is also demonstrated in a prospective cohort study, where at least $13 \%$ of injured runners faced a time to recovery exceeding 1 year. ${ }^{11}$ Furthermore, a systematic review revealed a yearly injury incidence rate of $21.6 \%-55.0 \%$ among recreational runners. ${ }^{12}$ 
Preventing running-related injuries should be considered a healthcare priority. ${ }^{13}$ Unfortunately, the evidence base for preventive interventions is nearly non-existent. ${ }^{14} 15$ A reason for this could be the limited knowledge about and insight into the aetiology behind running-related injuries, since preventive interventions build on insights into the mechanisms leading to injury. ${ }^{1617}$ For the past 20 years, it has been widely accepted that the majority of all running-related injuries are sustained as a consequence of repetitive structural overloading. ${ }^{18}$ Focus has too often been on one underlying mechanism (eg, only biomechanics or anthropometrics) or even on single variables within one of these mechanisms. To fully understand the aetiology of running-related injuries, it is of great importance to move away from this reductionist, monodisciplinary approach ${ }^{19}$ and be aware of its multifactorial nature. ${ }^{20}$ This entails considering biomechanical, clinical/anthropometrical and training-specific factors and their combined effects in the developmental process of running-related injuries. The non-training-related variables (eg, biomechanical variables and anthropometric variables) themselves cannot cause injury; the necessary cause is running. ${ }^{21}$ Nevertheless, the development and success of prevention strategies in running depend on understanding and targeting the underlying mechanisms and not only on modifying the training programme with regard to training load. Recently, causal frameworks have surfaced that introduce training load as a central and necessary part of the causal path to injury. ${ }^{21-23}$ In addition, changes in training load have been used as a primary exposure variable for injury across many sports. ${ }^{24-26}$

Changes in training load can be calculated in several ways. For instance, biweekly changes have been used as exposure in previous studies on running-related injury. ${ }^{26}{ }^{27}$ More recently, acute:chronic workload ratio (ACWR) was suggested as an alternative method to calculate changes in training load. ${ }^{28} \mathrm{ACWR}$ represents the ratio between the acute load (AL) (the load of the present week) divided by the chronic load (the average load of the present week and previous 3 weeks). In sports other than running, the association between ACWR and sports injury revealed a ratio $\geq 1.5$ associated with increased injury risk compared with a ratio in the so-called sweet spot ranging from 0.8 to $1.3 .^{28}{ }^{29}$ Although the classic method has advantages, four modifications should be considered. These include (1) using more than three exposure categories (zones), (2) reconsidering the method of calculating the ratio, (3) considering exponentially rolling averages and (4) including a measure of intensity, for example, rate of perceived exertion (RPE). The first modification is valuable for identifying doseresponse relationships. Categorising ACWR into three zones can identify a high-risk zone, but it is unsuitable for identifying whether there is a dose-response relationship or a U-shaped pattern. Presumably, the risk of injury increases exponentially as the ACWR rises. There are potentially three zones of low, moderate and highly increased risk. As a second modification, the method of calculating the ACWR is somewhat suspect, since the training volume from the present week is included both in the numerator and in the denominator of the equation. ${ }^{30}$ An alternative method of calculating the ACWR was presented by Malisoux et al, ${ }^{31}$ where the AL (present week) was not part of the chronic load (previous 4 weeks). Recently, this method of calculating changes in training load was used for handball. ${ }^{25} \mathrm{~A}$ third modification to the classic ACWR is to use an exponentially weighted moving average. Here, the weighting of the weekly training load decreases the further it moves away from the acute phase. ${ }^{32}$ As a final modification to address the issue of estimating internal load, it has been suggested to multiply RPE by a measure of distance or time. ${ }^{33}$ A combination of external (distance) and internal (intensity) load estimation would be necessary to understand both the work completed and the individual response. To date, ACWR, calculated in the classic way or in a modified way, has not been used as exposure to running-related injuries in any scientific study. This highlights the need to shed light on the ACWR and running-related injury development.

Effect measure modification is an analytical approach suitable for examining whether the difference in injury risk between low, moderate and high ACWR differs across subgroups of runners. ${ }^{22} 23$ The role of effect measure modifiers on changes in training load is rarely examined in sports injury literature ${ }^{34}$ despite its introduction in a sports injury setting more than two decades ago. ${ }^{35}$ The traditional analytical approach in sports injury research is instead to treat non-training variables as confounders, which has its disadvantages. For example, certain subgroups of runners who are particularly vulnerable to injury, such as those who suddenly change characteristics unrelated to training load, may be consequently overlooked using this approach. Effect measure modification has the potential to document such relationships. ${ }^{2022}$ This highlights the need for combining the modified version of ACWR (mACWR) and the concept of effect measure modification when addressing running-related injury thematic. To our knowledge, no study has used a modified version of the ACWR as primary exposure to running-related injury and included other time-varying or time-fixed variables as effect measure modifiers on this association.

\section{Purpose}

The main purpose of this study is threefold: (1) to investigate whether a U-shaped pattern exists for the association between mACWR and running-related injury (H1); (2) to investigate whether this U-shaped pattern is different between runners with different characteristics $(\mathrm{H} 2+\mathrm{H} 3)$; and (3) to investigate whether changes in mACWR (transitions between states) are associated with running-related injuries (H4). The secondary purpose of this study is to explore whether runners with between-leg differences in biomechanical and clinical/anthropometrical measures have increased risk of developing 
running-related injury compared with runners with no between-leg differences (H5).

\section{Hypotheses}

H1: after 52 weeks, runners with an mACWR between 0.8 and 1.3 will have the lowest injury risk of $5 \%$, whereas $2 \%, 5 \%$ and $10 \%$ more runners will sustain injuries in the $<0.8$ group, $1.3-1.7$ group and $>1.7$ group, respectively.

H2: after 52 weeks and using MACWR $0.8-1.3$ as a reference, the association between a moderate increase in mACWR (0.8-1.3 vs $1.3-1.7)$ and running-related injury will reveal an absolute difference of $10 \%$ if runners change running shoes, and no difference if they run in the same shoes. If the ratio exceeds 1.7 , there is no exacerbation caused by running shoes in the association between mACWR and running-related injury.

H3: after 52 weeks and using mACWR 0.8 to $1.3 \mathrm{mACWR}$ as a reference, the association between a moderate increase in mACWR (0.8-1.3 vs 1.3-1.7) and running-related injury will reveal an absolute difference of $10 \%$ if runners change surface, and no difference if they run on the same surface. If the ratio exceeds 1.7 , there is no exacerbation caused by running surface in the association between the mACWR and running-related injury.

H4: compared with the reference group (ACWR 0.8-1.3 across two states), $20 \%$ more runners will sustain an injury if they maintain a high ACWR $(>1.7$ across two states) or increase mACWR (transit from $<0.8$ to $>1.7$ or transit from 0.8 to 1.3 to $>1.7$ across two states) during the first 52 weeks.

H5: runners with a difference of at least \pm 1 SD in biomechanical or clinical/anthropometrical measures have an increased risk of injury compared with runners within the $68 \%$ prediction limit.

\section{METHODS}

Study protocol of a 52 week Prospective Running INjury study in Gothenburg (SPRING) is a 52-week prospective cohort study conducted at the Center for Health and Performance in Gothenburg, Sweden. All included subjects will provide written informed consent prior to inclusion according to the Swedish data protection agency. All reporting of scientific articles from this

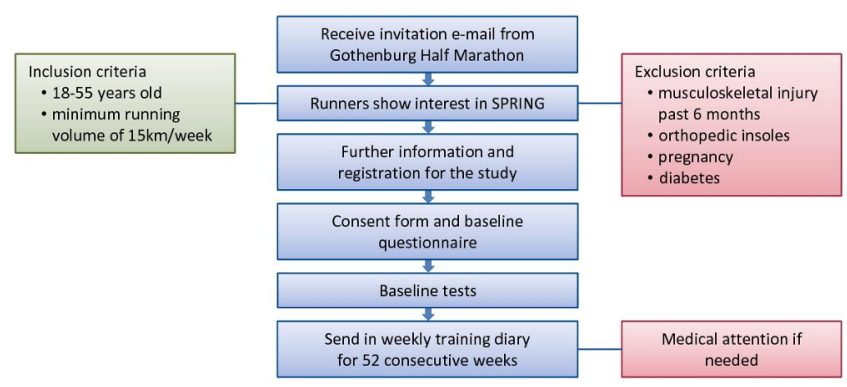

Figure 1 Flow chart of study procedure. SPRING, Study protocol of a 52-week Prospective Running INjury study in Gothenburg. research project will follow the STROBE statement. ${ }^{36} \mathrm{~A}$ flow chart of study procedure is presented in figure 1 .

\section{Study population}

The target population comprises healthy adult recreational adult runners. Healthy is defined as a person who has not suffered from any musculoskeletal injury in the lower extremities during the past 6 months. Recreational runners are defined as persons with an average weekly running volume of at least $15 \mathrm{~km}$ for the past 12 months prior to the baseline examination. Adult runners are defined as persons between the ages of 18 years and 55 years at the time of entering the study. Since degenerative changes in the properties of tendons, ligaments and bones are more likely to occur after the age of 55 years, this was chosen as the upper age limit. ${ }^{37}$

Participants will be recruited with the assistance of the Gothenburg Athletic Association, which organises the Gothenburg Half Marathon, the largest half-marathon in the world. This organisation keeps records (including email addresses) of runners who participated in the half-marathon the previous year. A subsample of runners from the Gothenburg area will be drawn randomly from these records, and an email will be distributed by Gothenburg Athletic Association to this subsample with an invitation to participate in the SPRING study. Runners responding to this invitation will receive a letter providing detailed information about the study purpose and methods, significance, inclusion/exclusion criteria, confidentiality and their right to withdraw from the study at any time from the researcher. If the inclusion/exclusion criteria are fulfilled, and the runner accepts the outlined information, the runner will receive an invitation for a baseline examination. The following additional exclusion criteria besides age, previous injury and weekly running distance are set: (1) participants are not allowed to use orthopaedic insoles due to the potential change in load distribution if they suddenly are removed, (2) pregnancy and (3) diabetes.

At the baseline examination, participants will be instructed to maintain their regular training habits after enrolment in the study and to log their weekly training in a specific training diary (online supplementary file 1) during the course of the study. The participants will fill in a questionnaire (online supplementary file 2) regarding their training habits and running experience and undergo anthropometric and clinical assessments and biomechanical measurements. These tests will be further described under effect-measure modifiers.

\section{Outcome}

The primary outcome measure is any running-related injury, which is defined as:

a running-related musculoskeletal pain in the lower limbs or back that causes a restriction on or stoppage of running (distance, speed, duration or training) in more than $66 \%$ of all training sessions in two consecutive weeks or in more than $50 \%$ of all 
training sessions in four consecutive weeks, or that requires the runner to consult a physician or other health professional which was modified from the consensus statement by Yamato et $a l^{38}$.

If a runner is injured during the study, he/she will be asked to attend a medical examination as soon as possible if the above-mentioned definition is fulfilled. In addition, an examination could also be performed if a participant requests an examination for a pain-related reason without fulfilling the above-mentioned standing criteria (non-running-related musculoskeletal injuries/ pain). All medical examinations will be carried out by the same medical practitioner, who has more than 30 years of experience. Subsequently, a detailed anamnesis, diagnosis and treatment plan will be summarised (online supplementary file 3 ). The runner will also be asked to report the first day of pain-free running after injury.

\section{Exposure}

Exposure for $\mathrm{H} 1-\mathrm{H} 4$

The primary exposure is mACWR. mACWR is the ratio of AL and weighted chronic loads (WCLs). AL is calculated as the running distance $(\mathrm{km})$ multiplied by the RPE for the present training session. Distance will be based on a self-reported number. RPE will be reported on a scale from 6 to $20 .{ }^{39} \mathrm{WCL}$ is the weighted sum of each AL the previous 28 days. The weighting is exponential, as described by Williams $e t a l,{ }^{32}$ where the older training loads are given less weight than the more recent loads. The exponential time decay is given by $2 /(N+1)$, where $N$ is time difference in days from the present training day. The WCL is calculated in formula 1.

$$
\sum_{N=1}^{28} \frac{2}{(N+1)}
$$

Thus, mACWR is calculated by:

$$
\frac{\mathrm{AL}}{\text { WCL1 + WCL2 . . WCL28 }}
$$

Since the primary exposure is considered time-varying in nature, each runner has the possibility to transit between exposure states during the period of data gathering. The cut-off values to separate exposure states will be $0.8,1.3$ and 1.7.

\section{Exposure for $\mathrm{H} 5$}

For the explorative examination, we will use a $68 \%$ prediction limit for all continuous risk factors. Cut-off values will be $\pm 1 \mathrm{SD}$; all values inside of that range will be considered normal (similar level of measure), and all values outside of that range will be considered as outside of normal range.

\section{Effect measure modifiers}

Since quantification of structure-specific load and structure-specific load capacity might be impossible to measure in epidemiological studies on running-related injuries, load distribution, load magnitude and load capacity-related variables will be measured instead. ${ }^{22}$ Measured variables are considered as time fixed, that is,

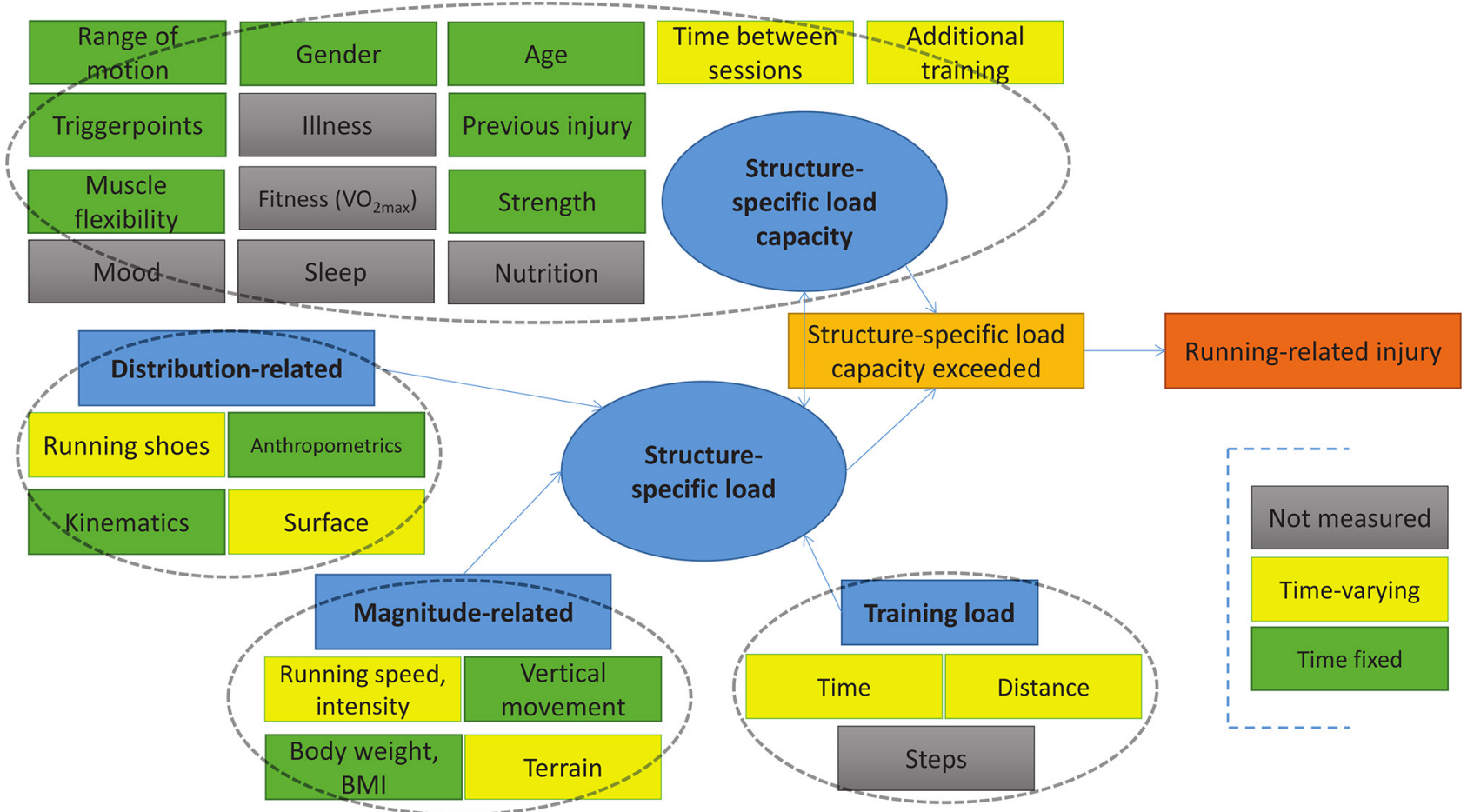

Figure 2 A directed acyclic graph-inspired approach to visualise the relationship between structure-specific load and structure-specific load capacity and running-related injury. Modified by a version from Bertelsen et al. ${ }^{22} \mathrm{BMI}$, body mass index. 
measured once at baseline examination, or time varying, that is. measured continuously every training session and reported on a weekly basis. A directed acyclic graph (DAG)-inspired diagram is presented in figure 2, describing the variables to be measured as well as those not to be measured, and if they are considered time fixed or time varying. According to DAG theory, distribution, magnitude-related and capacity-related variables should serve as effect measure modifiers on the association between changes in training load and running-related injuries. Even if these variables are not directly (causally) associated with the outcome, they may serve as risk factors on how much the subject could be exposed to running before experiencing the outcome (injury). In a running situation, this means that, for example, type of running shoes or amount of hip adduction (effect measure modifier) itself does not determine whether a runner will sustain an injury (outcome) but could influence how much change in running load (eg, ACWR exposure) the runner can tolerate before sustaining an injury.

\section{Distribution-related variables}

Distribution-related variables are movement patterns, anthropometric information, surface and type of running shoes.

\section{Movement patterns}

To describe movement patterns, all participants will run barefoot at a controlled speed of $12 \mathrm{~km} /$ hour $( \pm 5 \%)$ on a $12 \mathrm{~m}$ ethylene vinyl acetat foam runway in the laboratory. Sufficient time will be allowed for the subjects to get used to the laboratory, running surface and speed, enabling a habitual running style. A minimum of 25 running trials will be recorded for each subject using Qualisys Track Manager (QTM), a 16- camera infrared system (Qualisys AB, Gothenburg, Sweden) with a sampling frequency of $400 \mathrm{~Hz}$. The applied marker set consists of 32 spherical markers according to the International Society of Biomechanics recommendations. ${ }^{40}$ Using the same setup, except placing markers on the shoe instead of on the foot, subjects will then run with their own running shoes on the floor to compare barefoot and shod conditions.

Movement variables of interest are hip adduction range of motion (RoM), hip adduction velocity, knee flexion RoM, knee flexion velocity, rear foot pronation RoM, rear foot pronation velocity, ankle plantarflexion/ dorsiflexion RoM and sagittal touch down angle ground to foot. Motions of the hip, knee and ankle joints during stance phase will be calculated relative to the neutral standing position. Movement variables are considered as time fixed.

\section{Anthropometric information}

Anthropometric information includes segment lengths of femur, tibia and foot and will be estimated using QTM. Anthropometric variables are considered as time fixed.

\section{Surface}

Surface will be distinguished as asphalt, tartan, treadmill, gravel, grass, forest or 'other'. If a subject reports 'other' surface, they will have to specify in text. Surface is reported in percentage of the total distance of a running session and considered a time-varying variable.

Type of running shoe

Brand and model of running shoe will be reported for each training session and considered a time-varying variable.

\section{Magnitude-related variables}

Magnitude-related variables are vertical movement, body mass index (BMI) and terrain.

\section{Vertical movement}

Vertical movement is the vertical oscillation of the pelvis and will be calculated from the kinematic measurements. Vertical movement is considered time fixed.

\section{$\mathrm{BMI}$}

Body height will be determined to the nearest $0.5 \mathrm{~cm}$ by a ruler, and body weight will be determined using a calibrated personal scale (Kern MPB300K100; Balingen, Germany) to the nearest $0.1 \mathrm{~kg}$. BMI will be calculated from body height and body weight and considered a time-fixed variable.

\section{Terrain}

Terrain will be classified by the runner as uphill, downhill or flat. The participants will report this information in the weekly training diary in percentage of total distance. Terrain is considered time varying.

\section{Capacity-related variables}

Capacity-related variables are joint RoM, muscle flexibility, trigger points, isometric maximum strength, previous injury, gender, age, resting time between training sessions, stretching prior to or after a running session and additional training (other than running).

\section{Joint RoM}

Passive RoM measurements will be conducted in the different joints of the lower extremities and will be compared with standard values according to the neutralzero method ${ }^{41}$ to determine whether joint RoM is normal, restricted or excessive. The following standards of RoM assessment are defined as normal: hip flexion: $130^{\circ}-$ $140^{\circ}$; hip extension: $10^{\circ}-20^{\circ}$; hip abduction: $50^{\circ}-80^{\circ}$; hip adduction: $20^{\circ}-30^{\circ}$; hip internal rotation: $30^{\circ}-40^{\circ}$; hip external rotation: $40^{\circ}-50^{\circ}$; knee flexion: $120^{\circ}-150^{\circ}$; knee extension: $0^{\circ}-10^{\circ}$; ankle dorsiflexion: $10^{\circ}-20^{\circ}$; and ankle plantar flexion: $40^{\circ}-50^{\circ}$. RoM is classified as restricted or excessive when there is a visual difference of at least $\pm 10^{\circ}$ from normal. Joint RoM is considered a time-fixed variable. 
Muscle flexibility

Muscle flexibility will be visually assessed unilaterally for hamstrings and hip flexors ( $\mathrm{m}$. iliopsoas and $\mathrm{m}$. rectus femoris) and will be classified as normal, slightly restricted or clearly restricted. The following standards are defined as normal/slightly restricted/clearly restricted: hamstrings: $\geq 90^{\circ} / 70^{\circ}-90^{\circ} / \leq 70^{\circ}$; m. iliopsoas: $0^{\circ} / 0^{\circ}-20^{\circ} / \geq 20^{\circ} ; \mathrm{m}$. rectus femoris: $90^{\circ} / 70^{\circ}-90^{\circ} / \leq 70^{\circ}$, respectively. Muscle flexibility is considered a time-fixed variable.

\section{Trigger points}

Trigger points will be assessed at the tractus iliotibialis, $\mathrm{m}$. gastrocnemius, $\mathrm{m}$. soleus, $\mathrm{m}$. tibialis anterior, $\mathrm{m}$. tibialis posterior, m. gluteus medius and m. piriformis. Participants will be asked to inform the examiner whether the trigger points are accompanied with pain or not. Trigger points are considered a time-fixed variable.

\section{Isometric maximum strength}

Isometric maximum strength measurements for core and lower extremity muscles will be performed on DAVID devices (David Health Solutions, Helsinki, Finland) according to a standardised testing protocol. The following maximum strength measures will be assessed: trunk extension, trunk flexion, trunk rotation, hip abduction, hip adduction, knee extension and knee flexion. The following strength balance measures (ratios) will be calculated: trunk flexion:extension, trunk rotation right:left, hip abduction:adduction, knee extension left:right, knee flexion left:right and knee flexion:extension. Trunk flexion will be tested at $0^{\circ}$, trunk extension at $30^{\circ}$ and trunk rotation at $\pm 30^{\circ}$ (left and right sides). Hip abduction and adduction will be tested bilaterally in a hip abduction angle of $30^{\circ}$. Knee flexion and extension will be tested unilaterally at $30^{\circ}$ for knee flexion and $60^{\circ}$ for knee extension. All subjects will be seated and secured with a seatbelt, and they will not be allowed to self-stabilise during the measurement using their hands. During a short familiarisation period, subjects will be allowed to become accustomed to the test by first performing a dynamic movement against an increasing resistance, and then by performing submaximal isometric contractions. Participants will subsequently perform two maximum isometric contractions with a resting period of at least $30 \mathrm{~s}$, and the maximum torque value will be recorded. If the difference between the two tests exceeds $10 \%$, a third measurement will be conducted. The test leader will use verbal encouragement to increase the likelihood of reaching the subjects maximum strength potential. Maximum strength measures will be normalised to body weight. All strength measures are considered time-fixed variables.

\section{Previous injury}

Previous injuries and surgeries will be documented by a physiotherapist before the clinical/anthropometrical examination by questioning the runner according to a standardised procedure (online supplementary file 4). Previous injury is considered a time-fixed variable.

\section{Recoveryt}

Recovery time between running sessions will be reported indirectly in the weekly training diary by not filling in any information for possible training days. Recovery is considered a time-varying variable.

\section{Stretching}

Stretching prior to or after a running session will be reported (yes/no) in the weekly training diary for the following muscle groups: quadriceps, hamstrings, hip abductors, hip adductors and calf muscles. Stretching is considered a time-fixed variable.

\section{Other physical training}

Additional physical training (other than running) is considered a time-varying variable and will be reported as type of activity and duration. Low physical activity, such as gardening or walking for transportation, will not be considered as other physical training. The participants will be informed to consider any physical activity that requires changing to sports clothing as additional physical training.

\section{Age and gender}

Age and gender are considered time-fixed variables and will be registered at baseline.

\section{Statistical analysis}

Time-to-event statistics (pseudo-observation method through a generalised linear regression model) will be used to analyse the association between mACWR and running-related injury (RRI) using days as the time scale. ${ }^{42}$ Primary data analysis will take place at time point 365 , with a supplementary analysis at time point 180 . Runners will be censored in case of: discontinuation of the running schedule due to lack of motivation and/or time, health problems and other personal concerns hindering further participation. Cumulative risk difference will be used as a measure of association. The cumulative risk differences between time-to-first-injury will be compared between states/groups within each exposure.

To study whether the association between mACWR and RRI can be modified and thereby differs across strata of different variables; surfaces and shoes will be included in complementary analyses as effect measure modifiers in accordance with the recommendations by Rothman $e t$ $a l^{43}$ All results of the stratified analyses will be presented as the cumulative risk differences with $95 \%$ CI between exposure groups and within strata of each effect measure modifier. When using the pseudo-observation method to estimate risk differences in stratified analyses examining sample sizes of 50 and above, Hansen $e t a l^{44}$ found that at least 10 events (injuries) are needed per variable to avoid violating the statistical assumptions for valid analysis. Inclusion of any modifying variable will be determined in accordance with the recommendations described by 
Hansen et $a l .{ }^{44}$ Cumulative risk difference will be used as a measure of association when analysing H5. Statistical analyses will be performed using STATA/SE V.15 or later versions of the software package. Differences will be considered statistically significant at $\mathrm{p} \leq 0.05$.

\section{DISCUSSION}

SPRING is the first study that aims to investigate the association between running-related injuries and changes in training load using an modified version of the ACWR. The impetus for this study is a combination of the high injury rates occurring in recreational running, the high popularity of running among several European countries, as well as a lack of studies with a more multidisciplinary approach to running-related injuries. Reducing the amount of running-related injuries will enable runners to continue being physically active. This might be of great importance from a public health perspective, since physical exercise reduces the risk of numerous lifestyle diseases and improves physical and mental well-being.

The primary exposure variable in this study, mACWR, has not been used in any study on running-related injuries. mACWR cannot be calculated until the 28th day after inclusion. Therefore, runners with less training information than 28 days will be excluded from further analysis. This is inevitable when using the ACWR as a measure of changes in training load, no matter which version is used. Other methods for calculating changes in training load, for example, biweekly changes, can be used as early as after 2 weeks of training. However, it is reasonable to presume that mACWR contains more valuable training information, since more days are considered. Compared with the classical way of calculating acute:chronic workload, the MACWR is more sophisticated with weighted chronic training loads. For instance, if a runner ran 10 $\mathrm{km}$ at a similar exertion level yesterday and 14 days ago, it is clear that yesterday's run will affect the current (acute) level of fatigue and fitness more than the run 14 days ago and should thus be given more weight. Calculating weighted chronic training loads instead of non-weighted chronic training loads should therefore be considered if the aim is to estimate workload ratios. In the future, it may be necessary to investigate different calculation methods for weighted chronic training loads.

In summary, there is very little evidence about the relationship(s) of specific training parameters and the development of running-related injuries. One main reason for this deficit is that single training variables and their relationship to running-related injuries are usually investigated, neglecting possible modifying effects of other variables. Additionally, methodological limitations measuring training variables may have biassed and influenced the outcome of previous studies. The current study could possibly be biased by its reliance on self-reported training data. Thus, the primary exposure is based on subjective information. GPS technology is a more precise tool to evaluate training load and might be a possible option in the future to avoid this limitation. ${ }^{4546}$
In contrast, obtaining reliable information and a diagnosis from a medical practitioner regarding the outcome, running-related injuries, is a major strength of this study. It is also important to mention that the current definition of a running-related injury, which is rather strict compared with a simpler time-loss definition, could have an effect on future results.

Most importantly, the current study aims to shed light on associations that are likely to be closer to causal associations than the non-causal associations examined in reductionist-related studies. ${ }^{47}$ Several previous studies are based on a retrospective design. This is problematic, as differences between healthy and injured runners can neither be specified as causes nor as consequences of an injury. Prospective cohort studies are considered essential in order to identify problems related to temporality and to determine inter-relationships between different risk factors leading to injury. Further reasons for the evidence dilemma include, but are not limited to, inconsistent definitions of injury, different populations of runners (novice, recreational or elite), no control group, small study populations that lead to statistical underpowering, different kinematic models to evaluate movement pattern and other differences in measurement methods.

The long-term aim and perspective of this study is to create evidence-based prevention guidelines and training programmes with the ultimate goal of eliminating the risk of developing running-related injuries. This will benefit the participants in this study and the running community, coaches, physiotherapists and other people engaged in running.

Contributors JJ drafted the manuscript together with RØN, with feedback from PD, JK and SG. SG initiated the research project and designed the trial protocol. $\mathrm{JK}$ is the medical practitioner in the project. All authors approved the final manuscript.

Funding The study is funded by Sten A Olssons Foundation for Research and Culture located in Gothenburg, Sweden.

Disclaimer The funder of the study had no role in planning the study design or writing this manuscript, and will have no role in data collection or data analysis.

Competing interests None declared.

Patient consent Not required.

Provenance and peer review Not commissioned; internally peer reviewed.

Open access This is an Open Access article distributed in accordance with the Creative Commons Attribution Non Commercial (CC BY-NC 4.0) license, which permits others to distribute, remix, adapt, build upon this work non-commercially, and license their derivative works on different terms, provided the original work is properly cited and the use is non-commercial. See: http://creativecommons.org/ licenses/by-nc/4.0/

(c) Article author(s) (or their employer(s) unless otherwise stated in the text of the article) 2018. All rights reserved. No commercial use is permitted unless otherwise expressly granted.

\section{REFERENCES}

1. Hulteen RM, Smith JJ, Morgan PJ, et al. Global participation in sport and leisure-time physical activities: a systematic review and metaanalysis. Prev Med 2017;95:14-25.

2. Association SA. Löparrapporten 2017. 2017.

3. Pilgaard M, Rask S. Danskernes motions-og sportsvaner 2016 : Idrættens Analyseinstitut, 2016. 
4. Preuss H, Alfs C, Ahlert G. Sport als wirtschaftsbranche: der sportkonsum privater haushalte in Deutschland. Germany: SpringerVerlag, 2012

5. Scheerder J, Breedveld K, Danchev A. Running across Europe. 1. London: Palgrave Macmillan UK, 2015.

6. Fridberg T. Sport and exercise in Denmark, Scandinavia and Europe. Sport Soc 2010;13:583-92.

7. Hespanhol Junior LC, Pillay JD, van Mechelen W, et al. Metaanalyses of the effects of habitual running on indices of health in physically inactive adults. Sports Med 2015;45:1455-68.

8. Lee DC, Pate RR, Lavie CJ, et al. Leisure-time running reduces all-cause and cardiovascular mortality risk. J Am Coll Cardiol 2014;64:472-81.

9. Lee DC, Brellenthin AG, Thompson PD, et al. Running as a key lifestyle medicine for longevity. Prog Cardiovasc Dis 2017:60:45-55.

10. Koplan JP, Rothenberg RB, Jones EL. The natural history of exercise: a 10-yr follow-up of a cohort of runners. Med Sci Sports Exerc 1995;27:1180-4.

11. Nielsen RO, Rønnow L, Rasmussen S, et al. A prospective study on time to recovery in 254 injured novice runners. PLoS One 2014:9:e99877.

12. Kluitenberg B, van Middelkoop M, Diercks R, et al. What are the differences in injury proportions between different populations of runners? A systematic review and meta-analysis. Sports Med 2015;45:1143-61.

13. Hespanhol Junior LC, van Mechelen W, Postuma E, et al. Health and economic burden of running-related injuries in runners training for an event: A prospective cohort study. Scand J Med Sci Sports 2016;26:1091-9.

14. Kozinc Ž, Šarabon N. Effectiveness of movement therapy interventions and training modifications for preventing running injuries: a meta-analysis of randomized controlled trials. J Sports Sci Med 2017;16:421.

15. Yeung EW, Yeung SS. A systematic review of interventions to prevent lower limb soft tissue running injuries. Br J Sports Med 2001;35:383-9.

16. Bahr R, Krosshaug T. Understanding injury mechanisms: a key component of preventing injuries in sport. Br J Sports Med 2005;39:324-9.

17. Drew MK, Cook J, Finch CF. Sports-related workload and injury risk: simply knowing the risks will not prevent injuries. Br J Sports Med 2016

18. Hreljac A, Etiology HA. Etiology, prevention, and early intervention of overuse injuries in runners: a biomechanical perspective. Phys Med Rehabil Clin N Am 2005;16:651-67.

19. Hulme A, Finch CF. From monocausality to systems thinking: a complementary and alternative conceptual approach for better understanding the development and prevention of sports injury. Inj Epidemiol 2015;2:31.

20. Meeuwisse WH, Tyreman $\mathrm{H}$, Hagel B, et al. A dynamic model of etiology in sport injury: the recursive nature of risk and causation. Clin J Sport Med 2007;17:215-9.

21. Malisoux L, Nielsen RO, Urhausen A, et al. A step towards understanding the mechanisms of running-related injuries. J Sci Med Sport 2015;18:523-8.

22. Bertelsen ML, Hulme A, Petersen J, et al. A framework for the etiology of running-related injuries. Scand J Med Sci Sports 2017:27:1170-80

23. Nielsen RO, Bertelsen ML, Møller M, et al. Training load and structure-specific load: applications for sport injury causality and data analyses. Br J Sports Med 2017:bjsports-2017-097838.

24. Gabbett TJ, Jenkins DG. Relationship between training load and injury in professional rugby league players. J Sci Med Sport 2011;14:204-9.

25. Møller M, Nielsen RO, Attermann J, et al. Handball load and shoulder injury rate: a 31-week cohort study of 679 elite youth handball players. Br J Sports Med 2017;51:231-7.
26. Nielsen RØ, Parner ET, Nohr EA, et al. Excessive progression in weekly running distance and risk of running-related injuries: an association which varies according to type of injury. J Orthop Sports Phys Ther 2014;44:739-47.

27. Buist I, Bredeweg SW, Lemmink KA, et al. Predictors of runningrelated injuries in novice runners enrolled in a systematic training program: a prospective cohort study. Am J Sports Med 2010;38:273-80.

28. Gabbett TJ. The training-injury prevention paradox: should athletes be training smarter and harder? Br J Sports Med 2016;50:bjsp orts-2015-095788.

29. Soligard T, Schwellnus M, Alonso JM, et al. How much is too much? (Part 1) International olympic committee consensus statement on load in sport and risk of injury. Br J Sports Med 2016;50:1030-41.

30. Lolli L, Batterham AM, Hawkins R, et al. Mathematical coupling causes spurious correlation within the conventional acute-tochronic workload ratio calculations. Br J Sports Med 2017:bjsp orts-2017-098110.

31. Malisoux L, Frisch A, Urhausen A, et al. Monitoring of sport participation and injury risk in young athletes. J Sci Med Sport 2013;16:504-8

32. Williams S, West $\mathrm{S}$, Cross MJ, et al. Better way to determine the acute:chronic workload ratio? Br J Sports Med 2017;51:209-10.

33. Bourdon PC, Cardinale M, Murray A, et al. Monitoring athlete training loads: consensus statement. Int J Sports Physiol Perform 2017;12(Suppl 2):S2-161-S2-170

34. Hulme A, Finch CF. The epistemic basis of distance running injury research: a historical perspective. J Sport Health Sci 2016;5:172-5.

35. Meeuwisse WH. Assessing causation in sport injury: a multifactoria model. Clin J Sport Med 1994;4.

36. Vandenbroucke JP, von Elm E, Altman DG, et al. Strengthening the Reporting of Observational Studies in Epidemiology (STROBE): explanation and elaboration. PLoS Med 2007;4:e297.

37. McCarthy MM, Hannafin JA. The mature athlete: aging tendon and ligament. Sports Health 2014;6:41-8.

38. Yamato TP, Saragiotto BT, Lopes AD. A consensus definition of running-related injury in recreational runners: a modified Delphi approach. J Orthop Sports Phys Ther 2015;45:375-80.

39. Borg G, Hassmén P, Lagerström M. Perceived exertion related to heart rate and blood lactate during arm and leg exercise. Eur J Appl Physiol Occup Physiol 1987;56:679-85.

40. Wu G, Siegler S, Allard P, et al. Standardization and Terminology Committee of the International Society of Biomechanics. ISB recommendation on definitions of joint coordinate system of various joints for the reporting of human joint motion--part I: ankle, hip, and spine. International Society of Biomechanics. J Biomech 2002;35:543-8.

41. Ryf C, Weymann A. The neutral zero method - a principle of measuring joint function. Injury 1995;26:1-11.

42. Parner ET, Andersen PK. Regression analysis of censored data using pseudo-observations. Stata Journal 2010;10:408.

43. Rothman KJ, Greenland S, Walker AM. Concepts of interaction. Am $J$ Epidemiol 1980;112:467-70.

44. Hansen SN, Andersen PK, Parner ET. Events per variable for risk differences and relative risks using pseudo-observations. Lifetime Data Anal 2014;20:584-98.

45. Dideriksen M, Soegaard C, Nielsen RO. Validity of self-reported running distance. J Strength Cond Res 2016;30:1592-6.

46. Nielsen RO, Cederholm P, Buist I, et al. Can GPS be used to detect deleterious progression in training volume among runners? $\mathrm{J}$ Strength Cond Res 2013;27:1471-8.

47. Stovitz SD, Verhagen E, Shrier I. Distinguishing between causal and non-causal associations: implications for sports medicine clinicians. Br J Sports Med 2017:bjsports-2017-098520. 